\title{
Bone Marrow-Derived Cells as a Therapeutic Approach to Optic Nerve Diseases
}

\author{
Louise A. Mesentier-Louro, ${ }^{1}$ Camila Zaverucha-do-Valle, ${ }^{2}$ \\ Paulo H. Rosado-de-Castro, ${ }^{3}$ Almir J. Silva-Junior, ${ }^{1}$ Pedro M. Pimentel-Coelho, ${ }^{1}$ \\ Rosalia Mendez-Otero, ${ }^{1}$ and Marcelo F. Santiago ${ }^{1}$ \\ ${ }^{1}$ Instituto de Biofísica Carlos Chagas Filho, Universidade Federal do Rio de Janeiro, 21941-902 Rio de Janeiro, RJ, Brazil \\ ${ }^{2}$ Instituto Nacional de Infectologia Evandro Chagas (INI), Fundação Oswaldo Cruz, 21040-900 Rio de Janeiro, RJ, Brazil \\ ${ }^{3}$ Instituto de Ciências Biomédicas, Universidade Federal do Rio de Janeiro, 21941-902 Rio de Janeiro, RJ, Brazil \\ Correspondence should be addressed to Louise A. Mesentier-Louro; lmesentier@biof.ufrj.br
}

Received 9 August 2015; Accepted 10 September 2015

Academic Editor: Armand Keating

Copyright (c) 2016 Louise A. Mesentier-Louro et al. This is an open access article distributed under the Creative Commons Attribution License, which permits unrestricted use, distribution, and reproduction in any medium, provided the original work is properly cited.

\begin{abstract}
Following optic nerve injury associated with acute or progressive diseases, retinal ganglion cells (RGCs) of adult mammals degenerate and undergo apoptosis. These diseases have limited therapeutic options, due to the low inherent capacity of RGCs to regenerate and due to the inhibitory milieu of the central nervous system. Among the numerous treatment approaches investigated to stimulate neuronal survival and axonal extension, cell transplantation emerges as a promising option. This review focuses on cell therapies with bone marrow mononuclear cells and bone marrow-derived mesenchymal stem cells, which have shown positive therapeutic effects in animal models of optic neuropathies. Different aspects of available preclinical studies are analyzed, including cell distribution, potential doses, routes of administration, and mechanisms of action. Finally, published and ongoing clinical trials are summarized.
\end{abstract}

\section{Introduction}

Optic neuropathy is an umbrella term encompassing a large number of disorders that cause optic nerve damage. The retrograde degeneration of axons of retinal ganglion cells (RGCs) within the optic nerve can ultimately lead to the death of RGCs, which have their cell bodies in the inner retina, culminating in irreversible visual loss [1]. Glaucoma, the leading cause of irreversible blindness worldwide, is a progressive neuropathy that results from mechanical axonal damage at the optic nerve head [2]. It has been estimated that 64.3 million people had glaucoma in 2013 and that this number will increase to 111.8 million in 2040 [3]. Although the etiology of glaucoma is still a matter of intense investigation, the following risk factors have been associated with the disease: elevated intraocular pressure, use of systemic or topical corticosteroids, advanced age, thinner central cornea, vascular dysregulation, myopia, larger optic disc, positive family history, and African or Afro-Caribbean origin. Currently, treatment of glaucoma is limited to medications and surgical or laser procedures that reduce intraocular pressure $[4,5]$.

In contrast to the progressive nature of glaucoma, acute optic neuropathies are characterized by the acute onset of visual loss and are usually caused by ischemia (ischemic optic neuropathies), traumatic brain injury (traumatic optic neuropathy), and infection or inflammation (optic neuritis). Other causes of optic nerve injury, with varied clinical presentations, are compression, toxic or nutritional causes, infiltration of neoplastic or inflammatory cells, and papilledema secondary to elevated intracranial pressure $[1,6,7]$. Optic neuropathy can also occur in hereditary neurodegenerative disorders related to primary mitochondrial dysfunction, as well as in two nonsyndromic mitochondrial hereditary optic neuropathies: Leber hereditary optic neuropathy and 
dominant optic atrophy. These two disorders have an estimated prevalence of 1:45,000 (in Europe) and 1:25,000 (in northern England), respectively [8-11]. Moreover, RGC death and optic nerve degeneration may occur in other highly prevalent neurological disorders, such as multiple sclerosis and Alzheimer's disease $[12,13]$.

After optic nerve injury, RGCs are unable to regenerate their axons and undergo apoptosis, mostly due to an intrinsic inability to regenerate but also due to the inhibitory environment of the central nervous system (CNS) $[14,15]$. In order to stimulate neuronal survival and axonal outgrowth, many groups have been working on animal models of glaucoma and optic nerve injury. Strategies to improve regeneration include attempts to shift the inhibitory environment of the CNS to a permissive one and to stimulate the intrinsic regenerative programs of RGCs. For instance, it has been shown that RGCs are able to grow their axons on peripheral nerve grafts [1618]. However, even though peripheral nerve grafting provides a permissive environment, it does not sustain RGC survival on a long-term basis after optic nerve transection [19].

More robust results have been obtained with the stimulation of RCG intrinsic regeneration program through, for example, the deletion of the phosphatase and tensin homolog (PTEN) or the suppressor of cytokine signaling 3 (SOCS3) [20-22]. After optic nerve injury, RGCs with deletion of both PTEN and SOCS3 have growing axons that form new synapses in the suprachiasmatic nucleus and reintegrate with the local circuitry [23]. Extensive regeneration has also been shown when adenoassociated virus (AAV) expressing short hairpin RNA against PTEN was coupled to AAV encoding ciliary neurotrophic factor (CNTF) and to a cyclic adenosine monophosphate (cAMP) analog [24]. The combination of PTEN deletion with the induction of inflammation through zymosan injection and elevation of intracellular cAMP has also led to long-distance regeneration and some evidence of functional recovery in this model $[25,26]$. Moreover, using quantitative proteomics, Belin and coworkers revealed a network of signaling hubs following optic nerve injury and identified $c-m y c$ as a key regulator of the intrinsic regenerative mechanisms of RGCs [27].

Although these approaches are very promising, they are not easily translated to the clinic. The development of novel molecular tools for gene silencing has created an exciting new field of research, but there is still a long way to go before promising findings are translated into approved therapies, mainly due to safety issues that must be resolved [28, 29].

Cell therapy has emerged as a promising tool in regenerative medicine. Different research groups have used embryonic stem cells or induced pluripotent stem cells to generate RGCs that could replace the lost cells [30-32]. Although these newly generated cells express RGC markers, after injection into the vitreous chamber most of the transplanted cells remain close to the injection site, showing little capacity to integrate into the retina [30].

Another line of investigation has indicated that bone marrow-derived cells, such as mesenchymal stem cells (BMMSCs) and mononuclear cells (BM-MNCs), could increase RGC survival and promote axonal regeneration after optic nerve injury in rodents [33-35]. In recent years, substantial experience has accumulated in the transplantation of BMMSCs and BM-MNCs in patients with neurological disorders, indicating the safety and the feasibility of this approach [36].

This review summarizes and discusses the main findings of preclinical studies that have investigated the therapeutic action of bone marrow-derived cells in animal models of optic neuropathies. The use of noninvasive imaging methods to assess the distribution of the transplanted cells in the visual system and to investigate the efficacy of cell therapies is also discussed.

\section{Bone Marrow Mononuclear Cells}

The therapeutic potential of BM-MNCs has been extensively investigated in several disorders, including acute myocardial infarction and stroke $[36,37]$. The mononuclear cell fraction is usually isolated from bone marrow aspirates by FicollPaque density gradient centrifugation. Alternative isolation methods include the use of Percoll density gradient centrifugation or the immunomagnetic depletion of polymorphonuclear cells and erythrocytes [38]. BM-MNCs comprise a heterogeneous population of cells with diverse functions, including hematopoietic stem cells (HSCs), hematopoietic progenitors, endothelial progenitor cells (EPCs), mesenchymal stem cells (MSCs), monocytes, and lymphocytes. The bone marrow not only produces several types of immune cells but also attracts and retains different types of leukocytes. Zhao et al. [39] have elegantly reviewed the cellular composition of bone marrow, with an emphasis on the trafficking of immune cells.

Pang and colleagues [40] have shown that HSCs represented less than $0.20 \%$ of BM-MNCs in young individuals, although they were more frequent in the bone marrow of older individuals. HSCs are able to self-renew and differentiate into lineage-restricted progenitors, which subsequently give rise to the different blood cells [41]. CD34 is an antigen expressed by human HSCs and hematopoietic progenitor cells. Although CD34 can be expressed by other cell types, such as EPCs [42] and monocytes [43], the expression of this antigen has been used for the isolation of a human bone marrow cell population enriched in HSCs [44].

EPCs are circulating bone marrow-derived cells involved in endothelial repair and postnatal angiogenesis, due to their capacity to differentiate into mature endothelial cells and to secrete soluble factors, such as insulin-like growth factor 1 (IGF-1) and vascular endothelial growth factor (VEGF). They express HSCs and endothelial cell markers and can be cultured and expanded in vitro after isolation [42, 45]. Interestingly, there is increasing evidence that endothelial dysfunction associated with an altered number of circulating EPCs might play a role in the pathogenesis of neurological disorders, such as Alzheimer's [46] and Parkinson's [47] diseases.

In addition, BM-MNCs contain $0.001 \%-0.01 \%$ mesenchymal stem cells (MSCs) that can be expanded in culture [48]. In view of the importance of MSCs for regenerative medicine, these cells are described in detail below. 


\section{Mesenchymal Stem Cells}

MSCs were first described by Friedenstein and coworkers [49], who observed fibroblast-like cells that adhered to plastic when bone marrow suspensions were plated. These cells were defined as colony-forming unit multipotent cells and were able to differentiate into adipocytes, chondrocytes, and osteoblasts [49].

Because they adhere to plastic, MSCs can be easily isolated by plating the mononuclear cell fraction or even the whole bone marrow suspension in tissue culture flasks. All contaminating nonadherent cells are removed after serial medium changes.

MSCs are characterized by the panel of positive and negative cell surface markers proposed by the International Society for Cellular Therapy in 2006 [50]. The MSC population is defined as $>95 \%$ positive for CD105, CD73, and CD90 and $>95 \%$ negative for CD $45, \mathrm{CD} 34, \mathrm{CD} 14$ or CD11b, CD79 $\alpha$ or CD19, and HLA-DR. As previously mentioned, MSCs must be adherent to plastic and must be able to differentiate into chondrocytes, adipocytes, and osteocytes. Other surface markers are also expressed by MSCs, such as CD44, CD166, Stro-1, CD106, and CD146 [51].

MSCs occupy anatomically distinct locations within the bone marrow and are also found in endosteal, stromal, and perivascular niches [52-54]. Physiologically, MSCs support the HSC niche, protecting HSCs from apoptotic stimuli and preventing their differentiation [55]. MSCs can also be found in other tissues, including adipose tissue, dental pulp, umbilical cord, and placenta [56].

MSCs have the capacity to migrate to sites of injury following their intravascular administration. This process depends on molecules present on the surface of MSCs and endothelial cells, such as P-selectin and integrins [57]. After adhering to the endothelium, MSCs are capable of crossing it in a metalloprotease-dependent manner [58].

Interestingly, MSCs are considered to be not inherently immunogenic, as they express low levels of HLA class I antigens and do not express, or express in negligible levels, HLA class II antigens as well as their costimulatory molecules such as CD80, CD86, and CD40 [59]. This characteristic allows their allogeneic transplantation, with little or no risk of rejection. Furthermore, MSCs can release several immunomodulatory mediators and can attract immune cells through the release of chemokines [60].

The current view is that MSCs can exert neuroprotective and proregenerative effects, mainly by secreting multiple factors that act in a paracrine fashion [61, 62]. These beneficial effects can be observed in animal models of several neurological disorders, including Huntington's disease [63], stroke [64-66], and epilepsy [67].

\section{Preclinical Studies}

Several studies have described the therapeutic effects of bone marrow-derived cells in animal models of optic nerve disease. The main characteristics and the principal findings of these preclinical studies are summarized in Table 1. Since MSCs can be found in several tissues, a few studies using sources other than bone marrow are included.

Bone marrow cells have been tested in several animal models of glaucoma. Yu and coworkers [69] injected rat BMMSCs intravitreally 2 weeks after the ligation of episcleral veins. They observed that more RGCs survived in the treated retinas and that these retinas expressed more basic fibroblast growth factor (bFGF) and CNTF [69].

Johnson et al. [71] and Harper et al. [70] used laser to cauterize the trabecular meshwork and injected BM-MSCs intravitreally. Both groups found a neuroprotective effect of BM-MSC transplantation. In addition, Harper has injected BM-MSCs that were engineered to secrete brain-derived neurotrophic factor (BDNF), which resulted in increased protection of RGCs. Importantly, these studies used different methods to estimate RGC survival and also used different doses and times of administration.

Emre and coworkers [68] increased the intraocular pressure in rats by injecting hyaluronic acid into the anterior chamber. One week after the induction, MSCs derived either from the bone marrow or from the adipose tissue were transplanted intravitreally. Retrogradely labeled RGCs were counted two and four weeks after cell transplantation, which showed that the number of RGCs was significantly increased at both time points in the treated animals compared to the untreated group. Furthermore, the authors found decreased levels of the cytokines interferon gamma (IFN- $\gamma$ ) and tumor necrosis factor alpha (TNF- $\alpha$ ) in the treated retinas.

BM-MSCs also protected RGCs in a model of glaucoma that used laser pulses directed to the eye in order to block the aqueous outflow [73]. Interestingly, when BM-MSCs were transplanted into the anterior chamber after the induction of open angle glaucoma-differently from all the abovementioned studies, in which BM-MSCs were injected into the vitreous body - the treatment improved the regeneration of the trabecular meshwork. This led to better control of the intraocular pressure and consequently to a decrease in RGC degeneration [74].

In a model of retinal ischemia and reperfusion, $\mathrm{Li}$ and coworkers [72] injected rat BM-MSCs into the vitreous body and found that the number of RGCs, compared to the untreated group, increased after 4 weeks. Treated retinas showed increased expression of bFGF, BDNF, and CTNF.

Several studies have used optic nerve crush or transection as a broad-spectrum model of diseases that affect the optic nerve. Our group, for instance, demonstrated that both rat BM-MNCs and BM-MSCs had therapeutic effects after optic nerve crush. Intravitreally injected BM-MNCs protected RGCs in the first 2 weeks (although the effect was lost at 4 weeks) and increased axonal outgrowth for at least 4 weeks after optic nerve crush. In addition, animals treated with BM-MNCs showed an increased expression of the immediate early gene NGFI-A in the superior colliculus after light stimulation, indicating full-length axonal regeneration and synaptic connection with neurons of the superior colliculus [34]. This finding was supported by the retrograde labeling of RGCs 2 months after optic nerve crush. DiI was injected in the SC of BM-MNC-treated and untreated animals, but only treated animals showed DiI-positive, exuberant RGCs in 


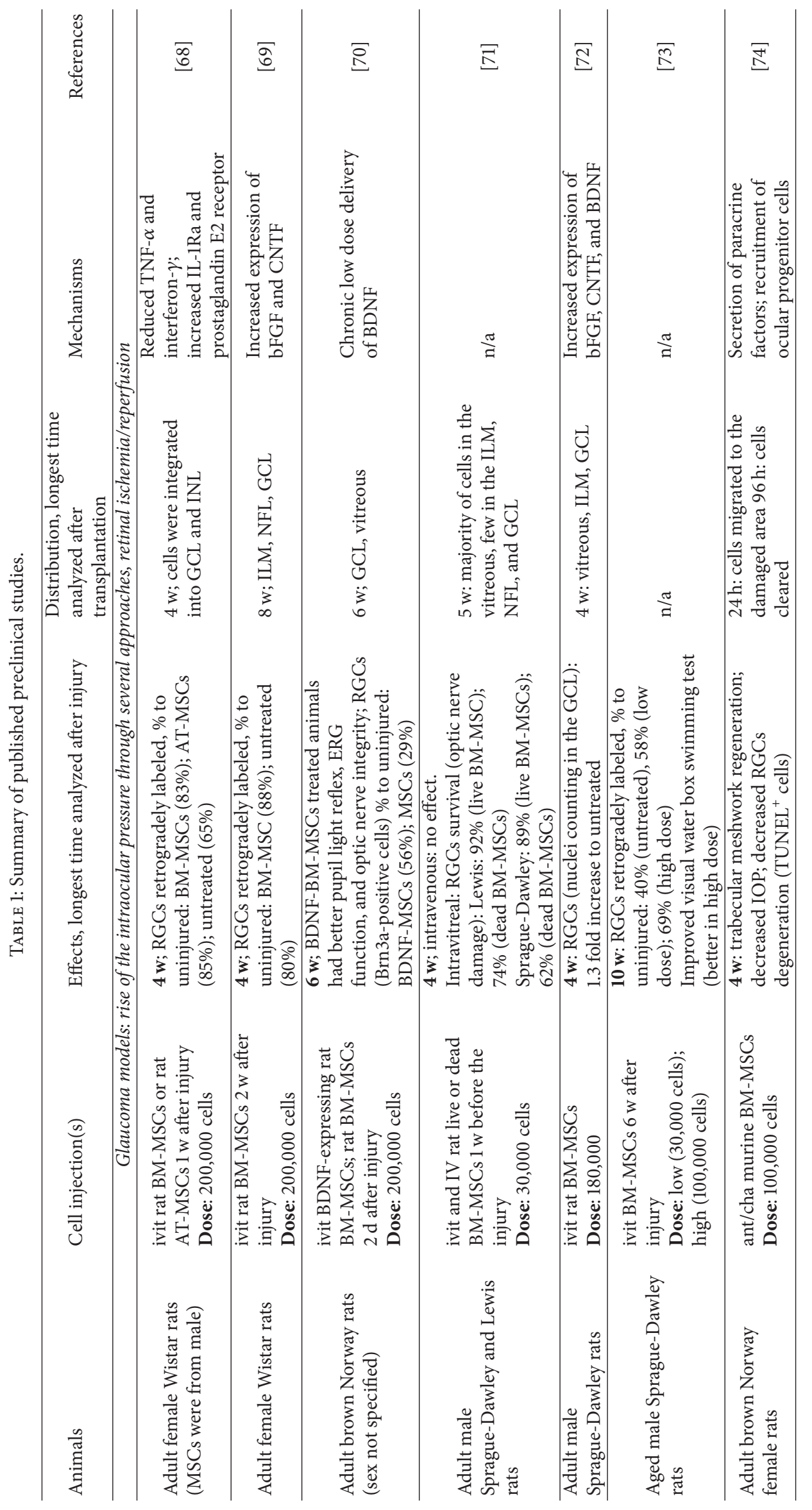




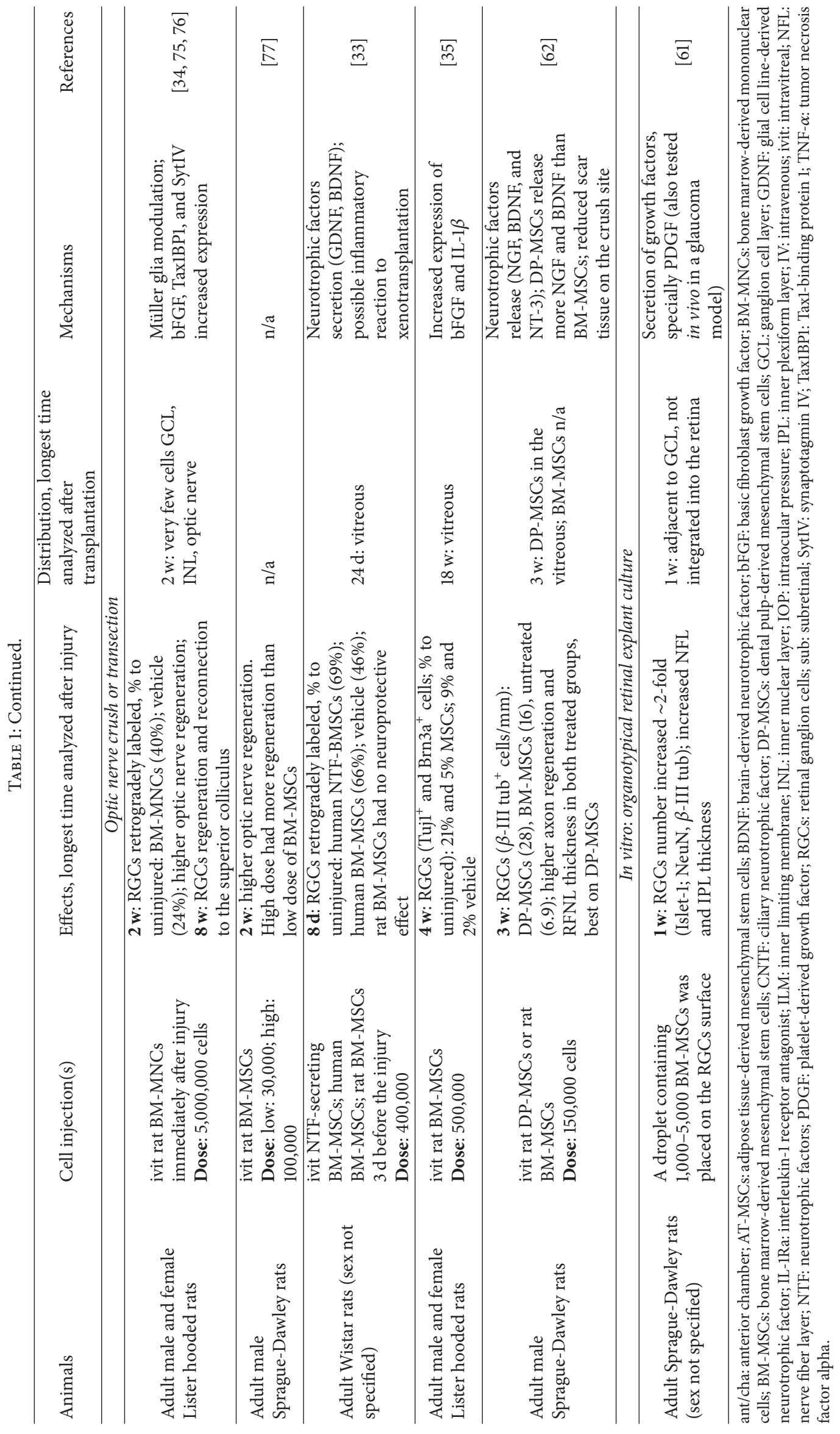


the retina. This finding indicated the complete regeneration of RGCs axons, allowing the transport of DiI from the axon terminal in the brain to the cell body in the retina [34].

On the other hand, in rats, intravitreal injection of BMMSCs led to sustained neuroprotection of RGCs for at least 4 weeks after optic nerve crush, which was the longest time period analyzed. Axonal outgrowth was also increased in these animals at the two time points analyzed ( 2 and 4 weeks). The association of sustained RGC survival and axonal regeneration in BM-MSC-treated animals suggests that RGCs may find favorable conditions to regenerate over longer distances and reconnect to their targets, as observed after BM-MNC treatment. This possibility is currently being investigated. Although BM-MNCs and BM-MSCs remained mostly in the vitreous body, treated animals showed increased expression of bFGF in their retinas. The antiapoptotic Taxl-binding protein 1 (Tax1BP1) and synaptotagmin IV gene expression were also upregulated in BM-MNCs treated retinas, while IL$1 \beta$ protein levels were increased in BM-MSCs-treated retinas $[34,35,75]$.

In another recent study, BM-MSCs were injected intravitreally after optic nerve crush and promoted an increase in axonal regeneration, in a dose-dependent manner [77]. An elegant study performed by Mead and coworkers [62] compared the effects of BM-MSCs with MSCs extracted from dental pulp (DP-MSCs), injected intravitreally after optic nerve crush. Although both cell populations were protective for RGCs and stimulated axonal regeneration, DPMSCs were more efficient. The authors showed that DPMSCs secreted larger amounts of nerve growth factor (NGF) and BDNF than BM-MSCs, indicating that trophic factor release determined the magnitude of the effect. Specific and unspecific blockage of Trk receptors A, B, or C significantly reduced the neuroprotective effect, suggesting a possible key role for neurotrophic factors that bind to this family of receptors, especially NGF, BDNF, and neurotrophin-3, in the effects of MSCs therapies [62].

MSCs derived from human umbilical cord blood (hUCBMSCs) were also tested as an alternative source. Zhao and coworkers injected hUCB-MSCs 7 days after optic nerve crush and observed improved RGC survival up to 28 days after the injury. They also observed increased levels of BDNF and glial cell line-derived neurotrophic factor (GDNF) in the retina after transplantation [78]. Similarly, Jiang and coworkers [79] transplanted hUCB-MSCs shortly after optic nerve crush and reported an increased survival of RCGs in treated animals. They also showed that treated animals had smaller decreases in amplitude and smaller increases in peak latency of the flash visual evoked potentials waveform compared to untreated animals and demonstrated the upregulation of GRP78 and downregulation of CHOP mRNA levels, suggesting that hUCB-MSCs could play a role in reducing endoplasmic reticulum stress [79].

Chen and coworkers, on the other hand, reported a transient effect of grafted hUCB-MSCs. Twenty-one days after injury, both RGC survival and GAP-43 expression increased in the retina of treated animals, but these differences were no longer present after 28 days [80].
A different perspective was provided by Johnson and coworkers [61], with a coculture model using retina and BM-MSCs. When retinas are removed from the eye to be cultured in vitro, RGC axons are axotomized and these cells progressively die. Coculture with BM-MSCs increased RGC survival, and analysis of the secretome of BM-MSCs indicated the presence of several growth factors, especially those of the platelet-derived growth factor (PDGF) family. Blocking of the PDGF signaling pathway abolished the neuroprotection conferred by BM-MSCs in the coculture system. Moreover, the intravitreal injection of PDGF homodimers (PDGF-AA) or heterodimers (PDGF-AB) after experimental elevation of the intraocular pressure reduced the degeneration of RGCs axons in the optic nerve [61].

In addition to MSCs, the bone marrow includes other cell types with potential therapeutic effects on the visual system. Monocytes represent a population of circulating bone marrow-derived cells that play important roles in vascular and tissue homeostasis, as well as in the responses to pathogens, toxins, and other types of insults [81, 82].

Monocytes are recruited to the ganglion cell layer and the inner plexiform layer, where they differentiate into macrophages, in the first days following retinal intoxication with glutamate, a murine model of RGC death. The intravenous injection of bone marrow-derived monocytes, 1 day

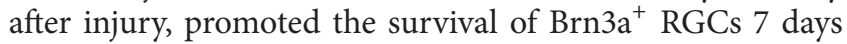
after the injury. This neuroprotective effect was further confirmed by Fluoro-Gold labeling of surviving RGCs. In contrast, the transfer of IL-10-deficient monocytes had no effect on RGC survival, indicating that the main mechanism of action of the transplanted monocytes was related to the release of this anti-inflammatory cytokine. In addition, transferred monocytes increased the number of proliferating neural progenitors in the ciliary body, although there was no evidence of neurogenesis [83].

A recent study, however, found that only $0.5-1 \%$ of the microglia/macrophages in the damaged retina came from circulating monocytes, 7-14 days after optic nerve transection in mice [84]. These findings suggest that the conditions for monocyte recruitment, such as alterations in the blood-brain barrier and the production of certain chemokines, might not be present in all types of optic neuropathies.

One strategy to stimulate optic nerve regeneration is the induction of intravitreal inflammation by injuring the lens or by intraocular injection of zymosan, a yeast cell wall preparation [85]. The proregenerative effect of zymosan seems to be mediated by the recruitment of neutrophils and macrophages (probably of monocyte origin) into the vitreous. Both cell types secrete oncomodulin, a growthpromoting factor for RGCs [86, 87]. However, Huang et al. [88] observed that whereas zymosan injection was able to improve the survival of RGCs after optic nerve axotomy in F344 rats, the treatment was detrimental to RGCs in a model of acute intraocular pressure elevation in this rat strain. Further investigation is therefore needed to elucidate the functional plasticity of monocytes [89] and the multifaceted role played by monocyte-derived macrophages in the visual system, under pathological conditions. 
4.1. Doses and Routes of Administration. Concerning the route of administration, most groups have injected the cells intravitreally. In one study, the systemic administration of BM-MSCs had no effect after experimental glaucoma induction, while the intravitreal injection protected RGCs and delayed axonal degeneration [71]. Interestingly, intravenously transplanted MSCs can be magnetically targeted to the retina. Yanai and coworkers labeled BM-MSCs with superparamagnetic iron oxide particles (SPIONs). The cells were injected into the tail vein and a gold-plated neodymium disc magnet was placed within the orbit to attract the cells to the eye [90]. This approach might represent an alternative to intravitreal or subretinal injections.

Cell doses were heterogeneous among the studies. Beneficial effects were observed with doses ranging from 30,000 to 500,000 BM-MSCs, while the BM-MNC dose used in our study was 5,000,000 cells (Table 1). Only a few studies have compared different doses of MSCs. In one study, the higher dose of MSCs was more efficient in improving the survival of RGCs, although the lower dose was also neuroprotective [73]. Similarly, another study found that a higher dose was more efficient in promoting optic nerve regeneration compared to a lower dose, although both doses were beneficial [77].

These observations suggest that the effects of MSCs are dose-dependent, but, to our knowledge, no studies have established the maximum tolerated dose for intraocular transplantation. There is a limit to the volume that can be injected into the vitreous body without causing damage to the eye, and therefore the number of injected MSCs cannot be too high.

Since we have observed that the neuroprotection conferred by BM-MNCs decays over time and that most of the transplanted cells are cleared from the vitreous body within 2 weeks, we investigated whether a second administration would change this outcome. We found that, even with a second dose, neuroprotection is lost over time. Axonal regeneration, however, was improved after the second injection, suggesting that BM-MNCs may provide neuroprotection and stimulate axonal outgrowth through different pathways [76].

4.2. Distribution and Persistence of the Transplanted Cells. In regenerative medicine, it is essential to determine where and how long the cells remain in the host tissue after transplantation and whether this phenomenon contributes to therapeutic effects.

Several studies have tracked bone marrow cells after injection in animal models of visual diseases. Yu and coworkers injected green fluorescent protein (GFP) expressing BMMSCs into the vitreous body, after inducing glaucoma by ligation of episcleral veins in adult rats. Two weeks after the injection, they found GFP-positive cells along the inner limiting membrane, with few of them integrated into the ganglion cell layer. Transplanted cells were found in the host tissue for up to 8 weeks.

Interestingly, $\mathrm{Na}$ and coworkers found that when BMMSCs were transplanted into normal eyes, they remained in the vitreous cavity. However, in ischemia/reperfusion injured retinas, BM-MSCs were found along the inner limiting membrane, and few of them were integrated into the ganglion cell layer, 4 weeks after injection [72].

In glaucomatous eyes, induced by photocoagulation of the trabecular meshwork, the majority of the BM-MSCs remained in the vitreous body, occasionally attached to the posterior lens capsule, and a small number of them reached the nerve fiber layer and the ganglion cell layer [71]. Harper and coworkers [70] described a similar profile of BM-MSC grafting in glaucomatous eyes, while Emre and coworkers [68] reported that BM-MSCs or adipose tissue-derived MSCs integrated into the ganglion cell layer and inner nuclear layer when the intraocular pressure was raised by injecting hyaluronic acid.

Johnson and coworkers [91] also developed in vitro coculture system to investigate the engraftment of BMMSCs in the retina. Similar to in vivo experiments, in this coculture system the BM-MSC grafts remained adjacent to the inner limiting membrane and did not integrate into the neural retina. In this study, the administration of a gliotoxic glutamate analogue enhanced BM-MSC grafting into the inner retinal layers, suggesting that glial cells are responsible for the poor integration of transplanted cells into the retina [91].

The use of histochemistry for cell tracking has many limitations. For example, misleading results can be obtained if the dye used to label transplanted cells is able to diffuse to retinal cells, as observed after labeling BM-MSCs with $4^{\prime}, 6$ diamidino-2-phenylindole (DAPI) [92, 93].

The use of noninvasive in vivo imaging methods may improve the understanding of numerous questions in the field of cell therapies for optic nerve diseases, including aspects such as the evaluation of cell distribution. Firstly, the retina may be examined through the clear cornea and lens by ophthalmoscopy [94]. Scanning laser ophthalmoscopy (SLO) employs laser light at a certain wavelength to scan the retina [95]. Different SLO equipment allows confocal SLO (cSLO), which has millimetric resolution and may be used to visualize graft size [94]. However, other methods are required to distinguish transplanted cells in the eye and any possible migration outside the eye. One of the possible methods is to transfect the cells with reporter genes for fluorescence imaging (FLI). This approach indicates the presence of cells that are metabolically active expressing the reporter gene [96]. Fischer and colleagues transfected human BMMSCs with a plasmid expression vector encoding enhanced GFP (eGFP) and incorporated the cells into miniaturized alginate spheres (MicroBeads) [97]. Then, the MicroBeads were subretinally implanted and tracked using cSLO [97]. The authors reported that eGFP-expressing cells encapsulated in MicroBeads continued to be viable for up to 4 months [97]. FLI may also be applied to macroscopically investigate if migration outside the eye takes place after intravitreal cell injection [98]. However, although the use of reporter genes is important for experimental investigations of cell migration, further studies are necessary to establish the safety of using viruses for transfection $[99,100]$.

Another method to macroscopically evaluate cell migration is to directly label cells with a radionuclide for nuclear medicine imaging or an exogenous contrast agent for MRI. 
Radionuclide cell labeling has been used for decades to diagnose infections through evaluation of the migration of labeled leukocytes by Single Photon Emission Computed Tomography (SPECT) [101]. More recently, SPECT has been applied for tracking cell therapies in different preclinical and clinical studies, with radionuclides such as Technetium-99m $\left({ }^{99 m} \mathrm{Tc}\right)$. PET may also be used for cell tracking, notably with fluorine-18 radiopharmaceuticals [96]. SPECT and PET may be acquired with conventional computed tomography (CT) and in combination as hybrid SPECT/CT or PET/CT. Such hybrid images permit simultaneous evaluation of functional and morphological information [102, 103]. Nevertheless, radiopharmaceutical cell labeling has restrictions, such as its relatively narrow time window for imaging. ${ }^{99 \mathrm{~m}} \mathrm{Tc}$, the most frequently used radionuclide, has a 6-hour half-life and permits imaging for approximately 24 hours [101].

Contrast agents for MRI may surmount many of the present limitations of radiopharmaceuticals. SPIONs were originally created as intravenous contrasts for hepatic imaging and more recently have been used by different groups for cell labeling. One advantage of SPIONs is that the iron in the particles can be tracked by MRI in vivo for several days or weeks and also ex vivo by histochemistry (e.g., prussian blue stain). In addition, SPIONs can be detected by immunofluorescence against a polysaccharide (dextran) used for particle coating. Nonetheless, SPIONs have limitations, such as the possibility of dilution after cell proliferation, as well as sequestration of the iron from dying cells by macrophages or microglia [104].

To combine the advantages of SPECT/CT and MRI cell tracking, our group has investigated the distribution of BMMNCs, using cell labeling with SPIONs (FeraTrack, Miltenyi Biotec, Germany) and ${ }^{99 \mathrm{~m}}$ Tc in a model of optic nerve crush [76]. Immediately after the lesion, $5 \times 10^{6}$ labeled cells or $5 \mu \mathrm{L}$ of saline was injected intravitreously. SPECT/CT was performed in the animals that received ${ }^{99 \mathrm{~m}}$ Tc-labeled BMMNCs, 1 hour after the cell transplant, and MRIs were acquired in animals that received SPION-labeled BM-MNCs up to 14 days after the cell therapy. Short-term tracking with SPECT/CT indicated that ${ }^{99 \mathrm{~m}}$ Tc-labeled BM-MNCs were restricted to the eye and did not enter the bloodstream after injection [76]. However, long-term tracking with MRI indicated that the signal from SPION-labeled BM-MNCs had decreased 5 days after intravitreal injection and was almost absent at 14 days [76]. This was confirmed after labeling BM-MNCs with a fluorescent dye, which indicated that the number of cells was already reduced at 3 days after the injection [76] and that the cells were absent after 14 days [75]. On the other hand, BM-MSCs labeled with SPIONs were found in vivo inside the eye for up to 18 weeks and were also observed ex vivo, found predominantly in the vitreous body [35].

Taken together, these results suggest that while BMMNCs injected into the vitreous body are cleared from this region within the first 2 weeks $[34,75]$, BM-MSCs can remain at the site for several weeks [35]. Despite their different temporal distributions, BM-MNCs and BM-MSCs were found mostly in the vitreous body, with poor integration into the inner retinal layers $[34,35]$. Interestingly, there was an important difference in the duration of therapeutic effects between BM-MNC and BM-MSC therapy: while the neuronal protection observed in BM-MNC-treated animals was lost from 2 to 4 weeks after injury [76], BM-MSCtreated animals showed increased neuronal survival for at least 4 weeks after injury and cell transplantation [35]. These findings suggest a possible correlation between the persistence of transplanted cells at the injection site and their beneficial effects.

Several studies suggest that bone marrow-derived cell grafts act in a paracrine fashion and that neurotrophic factors play a role in the therapeutic effect. It is possible that the paracrine effect would be enhanced if transplanted cells could remain longer in the damaged tissue, given the progressive nature of several optic neuropathies. However, the length of time that the presence of grafted cells is necessary to sustain neuronal survival and/or to stimulate regeneration remains to be investigated. It is also important to determine whether once these goals are achieved, the permanence of transplanted cells would somehow impair visual function by, for example, eliciting a sustained inflammatory response in the eye.

While intravitreally transplanted MSCs may remain for several months inside the eye, different results were observed when BM-MSCs were injected into the anterior chamber in a model of glaucoma. They were cleared from the tissue within 96 hours, probably phagocytosed by microglial cells, since about $20 \%$ of the transplanted cells expressed the microglia/macrophage marker F4/80 on day 2. In spite of the short time they remained, MSCs were able to induce the regeneration of the damaged trabecular meshwork [74]. In our experience, intravitreally injected MSCs remained in the vitreous body for more than 4 months without expressing the microglia/macrophage marker Ibal, although Ibal-positive cells were found in their vicinity [35]. It remains to be determined whether these differences could be attributed to the site of injection or if MSCs are able to remain in the damaged area only as long as they are needed to repair the tissue. Evidence against the latter hypothesis was provided by Haddad-Mashadrizeh and collaborators, who showed that adipose-derived MSCs injected into the vitreous cavity of the intact eye remained in the ocular tissue for up to six months, suggesting that these cells are not cleared from the tissue, even in the absence of an injury [105].

4.3. Evaluation of Structural Effects Using Noninvasive Methods. In addition to analyzing cell distribution, noninvasive imaging methods also allow the assessment of structural parameters that can be used in the investigation of the safety and efficacy of cell therapies. Fischer and coworkers used cSLO to study ocular integrity and evaluate possible modifications in the anatomy of the cornea, lens, vitreous, and retina after a subretinal injection of MicroBeads containing eGFPexpressing BM-MSCs [97]. The authors reported that when several MicroBeads per eye were implanted into the subretinal space, significant retinal detachment and disruption of retinal integrity were seen in cSLO [97]. Optical coherence tomography (OCT) is another powerful imaging method 
that employs light to acquire three-dimensional images and allows retinal and optic nerve evaluation at a micrometer resolution in preclinical and clinical settings [95]. Fischer and colleagues were able to detect single MicroBeads and demonstrate their structural integrity [97]. On the other hand, Mead and coworkers used OCT as a tool to study the efficacy of intravitreally injecting BM-MSCs or DP-MSCs in adult rat RGCs after optic nerve crush [106]. One, two, and three weeks after the lesion, OCT was carried out to quantify the retinal nerve fiber layer width as a measure of axonal atrophy [106]. The authors reported that DP-MSCs conserved retinal nerve fiber layer for up to 14 days after optic nerve crush, while BM-MSCs had no effect on this parameter [106].

MRI is an important tool for the evaluation of the CNS and may be performed with or without the use of contrast agents such as gadolinium-diethylenetriamine pentaacetic acid (Gd-DTPA) and manganese $\left(\mathrm{Mn}^{2+}\right)$ [107]. However, Gd-DTPA accumulates in regions of disrupted blood-brain barrier and frequently does not reveal noninflammatory diseases of single-fiber projections [108]. $\mathrm{Mn}^{+2}$-enhanced MRI (MEMRI) was developed to allow fiber tract imaging. $\mathrm{Mn}^{2+}$ is a calcium analog that is incorporated into neurons and is actively transported along preserved microtubules, whereas lesions stop its propagation [108]. Preclinical MEMRI of different CNS structures is possible after subcutaneous [109], intraperitoneal [110], intravenous [111], aerosolized [112], intravitreal [113], and topical [114] administration.

Haenold and collaborators [108] acquired high-resolution MEMRI by intravitreal injection of $\mathrm{MnCl}_{2}$ immediately after optic nerve crush in mice. They reported that axonal transport was reduced and interrupted proximal and distal to the nerve crush, respectively. Based on previous observations that unstimulated lesioned RGCs undergo limited fiber regeneration spontaneously [115], the authors carried out MEMRI and confirmed limited long-term regeneration one year after optic nerve crush [108]. To our knowledge, no studies with MEMRI have yet been carried out to evaluate the effects of bone marrow-derived cell therapies in optic nerve diseases. However, Mansergh et al. used MEMRI to evaluate the therapeutic potential of the intravitreal transplantation of retinal progenitor cells in a mouse model of Leber hereditary optic neuropathy [116]. The authors observed significant differences in signal intensity of MEMRI in animals treated with retinal progenitor cells at 1 and 3 months, indicating improvement after cell therapy [116].

However, the use of $\mathrm{MnCl}_{2}$ has limitations, because overexposure to $\mathrm{Mn}^{2+}$ causes neurological toxicity in humans and animals $[113,117]$. Thuen et al. reported that rat RGCs directly exposed to intravitreally injected $\mathrm{MnCl}_{2}$ die at dosages higher than $300 \mathrm{nmol}$ infused $\mathrm{Mn}^{2+}$ [113].

Diffusion tensor imaging (DTI) is another valuable MRI technique that maps the random motion of water molecules and reflects CNS microstructural integrity and pathology [118]. Zhang et al. evaluated DTI in mice and reported that it detected axonal injury as early as 6 hours after optic nerve crush [118]. Thuen and coworkers investigated the combined use of MEMRI and DTI to detect axonal injury in rats and to evaluate the regenerative potential of intravitreally transplanting a peripheral nerve graft in the optic nerve [119].
4.4. Evaluation of Functional Effects Using Noninvasive Methods. Different parameters may be used to evaluate the functional effects of cell therapies in animal models of optic nerve injury, including optokinetic response, pupil light reflex (PLR), electroretinography (ERG), and visual evoked potentials (VEPs).

Optokinetic response may be analyzed to quantify the capacity of distinguishing spatial frequency and contrast sensitivity in animal models of glaucoma [120]. Mansergh and collaborators used a video camera to quantify the animals' slow tracking movements in response to moving stripes in a chamber and beneficial effects were seen at 1 and 3 months after cell therapy with retinal progenitors in a mouse model of Leber hereditary optic neuropathy [116].

PLR may be analyzed by computerized pupillometry [70]. Harper and coworkers used PLR to investigate the therapeutic effect of intravitreal therapy with BM-MSCs engineered to express BDNF and GFP (BDNF-BM-MSCs) or just GFP (GFP-BM-MSCs) in a rat model of chronic ocular hypertension [70]. PLR evaluation indicated increased therapeutic potential for functional improvement in eyes treated with BDNF-BM-MSCs in comparison to GFP-BMMSCs at 42 days after the lesion. They also investigated the use of ERG, which measures the electrical response of the retina to visual stimuli with corneal electrodes $[70,121]$. The analysis indicated that retinal electrical activity was preserved at 20 and 40 days, in rats that received BDNF-BM-MSCs [70].

VEP analyzes occipital lobe brain wave potential after visual stimuli [121]. The VEP is carried out using electrodes positioned over the occipital area of both hemispheres, one eye at a time [121]. The elongation of P100 latency is a frequent abnormality observed in optic nerve dysfunction [121]. Zhang and colleagues evaluated the VEP in rats following intravitreal injection of human umbilical cord blood-derived cells or BDNF 7 days after optic nerve crush [122]. The authors reported that VEP detection scores showed greater peak voltages and shorter peak latencies in the treated groups in comparison to the control group, indicating functional improvement after cell therapy [122].

4.5. Mechanisms of Action of Cell Therapies. Although the initial studies of cell therapy suggested that bone marrow cells could differentiate into neuronal cells [123-126], it was later shown that these findings could be attributed to cell fusion, rapid disruption of the actin cytoskeleton, and/or cellular toxicity [127-129].

In most of the preclinical studies discussed in the previous sections, neuroprotection was attributed to a paracrine effect, and no evidence of neural transdifferentiation of MSCs has been shown. In this respect, the therapeutic effect of transplanted cells could be related to the capacity of MSCs to alter the microenvironment of the injured tissue, through the release of trophic factors and inflammatory mediators [130]. This paracrine effect may occur without the need for the integration of MSCs into the neural retina.

BM-MSCs can secrete a variety of trophic factors [131] and our group showed that rat bone marrow cells express the mRNAs for BDNF, bFGF, CNTF, VEGF, and transforming growth factor alpha (TGF- $\alpha$ ) even without stimulation [34]. 
Moreover, the expression of CNTF, GDNF, BDNF, bFGF, and hepatocyte growth factor alpha chain $(\mathrm{HGF} \alpha)$ was observed in GFP-positive BM-MSCs engrafted in glaucomatous retinas [69], suggesting that the paracrine effect may contribute to the cell therapy effect.

In order to increase this paracrine activity, a few studies have engineered the cells before transplantation. Intravitreally injected rat or human BM-MSCs that were stimulated to secrete neurotrophic factors were found in clusters between the lens and the retina and remained in the eye for at least 3 weeks after optic nerve transection. In this study, human BM-MSCs were more neuroprotective than rat BMMSCs, which is correlated with the higher secretion of BDNF and GDNF by human cells, although it is possible that a beneficial inflammatory reaction could have been elicited by the xenotransplant [33].

In addition to the release of neurotrophic factors that may act directly on damaged neurons, the interaction between transplanted cells and retinal glial cells cannot be ignored. Indeed, we have shown that BM-MNC transplantation was associated with a reduction in the expression of glial fibrillary acidic protein (GFAP) in radial glial processes throughout the retinal layers, which is a marker of Müller cell reactivity in response to injury [34]. On the other hand, a recent study used BM-MSCs and suggested that the transplantation of these cells increased glial reactivity in the retina [132]. The implication of cell therapies for retinal gliosis and the contribution of these changes to the observed therapeutic effects need further elucidation.

A large number of studies have demonstrated that BM-MSCs exert beneficial immunomodulatory effects by interacting with cells of the innate and adaptive immune system $[130,133]$. Furthermore, BM-MSCs constitutively express several innate immune sensors, such as toll-like receptors (TLRs). These receptors recognize many molecules expressed/released by pathogens or released upon tissue injury, being involved in the initiation and regulation of immune responses [134]. For instance, TLR4-primed MSCs (also called MSC1) exhibit a proinflammatory profile, while TLR3-primed MSCs (MSC2) adopt an anti-inflammatory phenotype [135].

Several studies have suggested that the crosstalk between MSC and the injury microenvironment leads to the secretion of soluble factors by these cells [136]. For example, upon stimulation by proinflammatory cytokines such as TNF$\alpha$ and IFN- $\gamma$, MSCs secrete immunosuppressive mediators, including prostaglandin E2 (PGE2), indoleamine 2,3dioxygenase (IDO), transforming growth factor beta-1 (TGF$\beta 1$ ), and IL-10 [130]. The interaction with immune cells also takes place in the CNS. For example, MSCs cocultured with lipopolysaccharide-activated microglia reduced the expression of TNF- $\alpha$, inducible nitric oxide synthase (iNOS), and oxidative-stress related proteins by microglia and augmented the expression of IL- $1 \beta$, CX3CR1, NURR1, and EP2. Interestingly, the authors showed that the functional changes in microglia were induced by the release of the chemokine CX3CL1 by MSCs, switching microglia from a neurotoxic to a neuroprotective phenotype [137].
Besides the secretion of soluble factors, it has recently been suggested that the therapeutic effect of BM-MSCs may be partially related to the release of extracellular vesicles. These vesicles, named "exosomes" or "microvesicles" according to their size, contain proteins, mRNA, and microRNA that can be transported from one cell to another [136]. Proteins present in BM-MSC extracellular vesicles include signaling molecules such as mitogen-activated protein kinase 1 (MAPK1), cell adhesion mediators such as fibronectin, and surface receptors such as PDGF receptor. Extracellular vesicles derived from MSCs also express regulatory molecules such as membrane-bound TFG- $\beta$, galectin- 1 , and programmed death ligand-1 [138].

Considering the biological function of proteins and microRNAs transported by extracellular vesicles, it would be interesting to evaluate the therapeutic potential of BM-MSC extracellular vesicles in animal models of optic neuropathies. In a model of stroke, for example, Xin and coworkers [139] showed that the secretion of microRNA 133b, mediated by extracellular vesicles from BM-MSCs, contributed to the beneficial effects of cell therapy. In this model, BM-MSC exosomes were transferred to neurons and astrocytes [139].

\section{Published Clinical Studies of Bone Marrow Cell Therapies for Optic Nerve Lesions}

Jonas et al. published a case report of autologous BMMNC therapy for a 43-year-old patient with advanced retinal and optic nerve atrophy due to diabetic retinopathy [140]. The authors performed an intravitreal injection of $0.5 \mathrm{~mL}$ containing $1.8 \times 10^{8} \mathrm{BM}-\mathrm{MNCs}$ and concluded that the procedure was feasible and safe [140].

Connick and coworkers [141] carried out an open-label phase 2a study in patients with secondary progressive multiple sclerosis affecting the visual pathways. A mean dose of 1.6 $\times 10^{6}$ autologous BM-MSCs was injected intravenously in 10 patients with clinical and electrophysiological confirmation of optic nerve injury. The authors reported that there were no serious adverse events and described an increase in visual acuity, visual evoked response latency, and optic nerve area.

\section{Ongoing Clinical Studies of Bone Marrow Cell Therapies for Optic Nerve Lesions}

At least three studies registered in clinicaltrials.gov have been designed to carry out bone marrow cell therapy for optic neuropathies. Weiss and collaborators, from a private clinic in Florida, USA, started an open-label study in August 2013 with an estimated completion date in August 2017, where 300 patients are expected to receive autologous BMMSCs (NCT01920867). Retrobulbar, subtenon, intravenous, intravitreal, and intraocular injections will be administered to patients with retinal disease, macular degeneration, hereditary retinal dystrophy, optic nerve disease, and glaucoma, respectively. The primary and secondary outcome measurements will be visual acuity and visual fields, respectively, and patients will be followed up for 12 months. 
De Paula and coworkers, from the University of São Paulo, Brazil, have designed an open-label, single-group phase 1 study to perform intravitreal autologous transplantation of $10^{6} \mathrm{BM}-\mathrm{MSC}$ in patients with retinal degeneration or primary open-angle glaucoma (NCT02330978). The reported that the start date was January 2014 and a total of 10 patients are programmed to be included, with an estimated study completion date of December 2016. Patients will be followed up for 6 months. Primary outcome measurements will be the type and severity of adverse effects. The secondary outcome measurements will be changes in visual acuity, visual field, OCT parameters, and RGC function as assessed by ERG.

Jamadar and colleagues, from Chaitanya Hospital in Pune, India, started in September 2014 an open-label, phase 1/phase 2 study (NCT01834079). A total of 24 patients with optic nerve atrophy will be enrolled to receive an intrathecal injection of $10^{8}$ autologous BM-MNCs per dose in three applications at intervals of 7 days. The estimated study completion date is July 2016 and patients will be followed up for 6 months. The primary outcome measurement will be reduction in degeneration of the optic nerve with improvement in vision. Secondary outcome measurements will be an increase in visual function and improvement in idiopathic intracranial hypertension.

Other trials of stem cell therapies for degenerative eye diseases, such as diabetic and ischemic retinopathy, which may secondarily affect the optic nerve, have been recently reviewed by Mead and collaborators [142].

\section{Conclusion}

Studies using different animal models of optic nerve injury, such as optic nerve compression or transection and elevation of the intraocular pressure, have shown that RGC degeneration can be reduced by intravitreal transplantation of BM-MSCs or BM-MNCs, which was the delivery method used most often. Doses varied among the studies, but few of them suggested that higher doses have increased therapeutic potential. Bone marrow-derived cell effects were mostly attributed to the release of soluble factors that can protect RGCs and/or modulate the inflammatory response in the retina. Interestingly, there is evidence of long-term persistence of BM-MSCs at the injection site, although the importance of this phenomenon remains to be elucidated. Such preclinical studies showing the neuroprotective and proregenerative effects of bone marrow-derived cells have encouraged the execution of phase 1 or phase 2 clinical trials for diseases that affect the optic nerve. Several trials are ongoing and a few have been concluded, indicating the feasibility and safety of intravitreal or intravenous administration of autologous bone marrow-derived cells. Most preclinical studies focused on morphological outcomes such as RGC survival and axonal outgrowth, but as these parameters were improved in treated animals, there is a growing need for visual functional analysis in future studies. Further investigations are also necessary to unravel the mechanisms of action of transplanted cells, in order to allow the development of safe and efficient therapies.

\section{Conflict of Interests}

The authors declare that there is no conflict of interests regarding the publication of this paper.

\section{Acknowledgments}

The authors are grateful for the financial support from Conselho Nacional de Desenvolvimento Científico e Tecnológico (CNPq), Fundação Carlos Chagas Filho de Amparo à Pesquisa do Estado do Rio de Janeiro (FAPERJ), Coordenação de Aperfeiçoamento de Pessoal de Nível Superior (CAPES), Programa Ciência Sem Fronteiras-CAPES, and Departamento de Ciência e Tecnologia do Ministério da Saúde (DECIT). The authors wish to thank Janet W. Reid for revising and editing the language in the text.

\section{References}

[1] A. Ghaffarieh and L. A. Levin, "Optic nerve disease and axon pathophysiology," International Review of Neurobiology, vol. 105, pp. 1-17, 2012.

[2] I. Soto and G. R. Howell, "The complex role of neuroinflammation in glaucoma," Cold Spring Harbor Perspectives in Medicine, vol. 4, no. 8, 2014.

[3] Y.-C. Tham, X. Li, T. Y. Wong, H. A. Quigley, T. Aung, and C.Y. Cheng, "Global prevalence of glaucoma and projections of glaucoma burden through 2040: a systematic review and metaanalysis," Ophthalmology, vol. 121, no. 11, pp. 2081-2090, 2014.

[4] M. Almasieh, A. M. Wilson, B. Morquette, J. L. Cueva Vargas, and A. Di Polo, "The molecular basis of retinal ganglion cell death in glaucoma," Progress in Retinal and Eye Research, vol. 31, no. 2, pp. 152-181, 2012.

[5] R. N. Weinreb, T. Aung, and F. A. Medeiros, "The pathophysiology and treatment of glaucoma: a review," The Journal of the American Medical Association, vol. 311, no. 18, pp. 1901-1911, 2014.

[6] R. Behbehani, "Clinical approach to optic neuropathies," linical Ophthalmology, vol. 1, no. 3, pp. 233-246, 2007.

[7] D. P. Dworak and J. Nichols, "A review of optic neuropathies," Disease-a-Month, vol. 60, no. 6, pp. 276-281, 2014.

[8] V. Carelli, C. La Morgia, M. L. Valentino, P. Barboni, F. N. RossCisneros, and A. A. Sadun, "Retinal ganglion cell neurodegeneration in mitochondrial inherited disorders," Biochimica et Biophysica Acta-Bioenergetics, vol. 1787, no. 5, pp. 518-528, 2009.

[9] C. La Morgia, M. Carbonelli, P. Barboni, A. A. Sadun, and V. Carelli, "Medical management of hereditary optic neuropathies," Frontiers in Neurology, vol. 5, article 141, 2014.

[10] B. Mascialino, M. Leinonen, and T. Meier, "Meta-analysis of the prevalence of Leber hereditary optic neuropathy mtDNA mutations in Europe," European Journal of Ophthalmology, vol. 22, no. 3, pp. 461-465, 2012.

[11] P. Yu-Wai-Man and P. F. Chinnery, "Dominant optic atrophy: novel OPA1 mutations and revised prevalence estimates," Ophthalmology, vol. 120, no. 8, p. 1712.el, 2013.

[12] H. V. Danesh-Meyer, H. Birch, J. Y.-F. Ku, S. Carroll, and G. Gamble, "Reduction of optic nerve fibers in patients with Alzheimer disease identified by laser imaging," Neurology, vol. 67, no. 10, pp. 1852-1854, 2006. 
[13] A. London, I. Benhar, and M. Schwartz, "The retina as a window to the brain-from eye research to CNS disorders," Nature Reviews Neurology, vol. 9, no. 1, pp. 44-53, 2013.

[14] M. E. Schwab, J. P. Kapfhammer, and C. E. Bandtlow, "Inhibitors of neurite growth," Annual Review of Neuroscience, vol. 16, pp. 565-595, 1993.

[15] V. Pernet and M. E. Schwab, "Lost in the jungle: new hurdles for optic nerve axon regeneration," Trends in Neurosciences, vol. 37, no. 7, pp. 381-387, 2014.

[16] A. J. Aguayo, M. Vidal-Sanz, M. P. Villegas-Perez, and G. M. Bray, "Growth and connectivity of axotomized retinal neurons in adult rats with optic nerves substituted by PNS grafts linking the eye and the midbrain," Annals of the New York Academy of Sciences, vol. 495, pp. 1-9, 1987.

[17] S. A. Keirstead, M. Vidal-Sanz, M. Rasminsky, A. J. Aguayo, M. Levesque, and K.-F. So, "Responses to light of retinal neurons regenerating axons into peripheral nerve grafts in the rat," Brain Research, vol. 359, no. 1-2, pp. 402-406, 1985.

[18] M. Vidal-Sanz, G. M. Bray, M. P. Villegas-Pérez, S. Thanos, and A. J. Aguayo, "Axonal regeneration and synapse formation in the superior colliculus by retinal ganglion cells in the adult rat," Journal of Neuroscience, vol. 7, no. 9, pp. 2894-2909, 1987.

[19] L.-P. Cen, J.-M. Luo, Y. Geng, M. Zhang, C. Pui Pang, and Q. Cui, "Long-term survival and axonal regeneration of retinal ganglion cells after optic nerve transection and a peripheral nerve graft," NeuroReport, vol. 23, no. 11, pp. 692-697, 2012.

[20] P. D. Smith, F. Sun, K. K. Park et al., "SOCS3 deletion promotes optic nerve regeneration in vivo," Neuron, vol. 64, no. 5, pp. 617623, 2009.

[21] F. Sun, K. K. Park, S. Belin et al., "Sustained axon regeneration induced by co-deletion of PTEN and SOCS3," Nature, vol. 480, no. 7377, pp. 372-375, 2011.

[22] K. K. Park, K. Liu, Y. Hu et al., "Promoting axon regeneration in the adult CNS by modulation of the PTEN/mTOR pathway," Science, vol. 322, no. 5903, pp. 963-966, 2008.

[23] S. Li, Q. He, H. Wang et al., "Injured adult retinal axons with Pten and Socs3 co-deletion reform active synapses with suprachiasmatic neurons," Neurobiology of Disease, vol. 73, pp. 366-376, 2015.

[24] B. J. Yungher, X. Luo, Y. Salgueiro, M. G. Blackmore, and K. K. Park, "Viral vector-based improvement of optic nerve regeneration: characterization of individual axons' growth patterns and synaptogenesis in a visual target," Gene Therapy, 2015.

[25] S. de Lima, Y. Koriyama, T. Kurimoto et al., "Full-length axon regeneration in the adult mouse optic nerve and partial recovery of simple visual behaviors," Proceedings of the National Academy of Sciences of the United States of America, vol. 109, no. 23, pp. 9149-9154, 2012.

[26] T. Kurimoto, Y. Yin, K. Omura et al., "Long-distance axon regeneration in the mature optic nerve: contributions of oncomodulin, cAMP, and pten gene deletion," The Journal of Neuroscience, vol. 30, no. 46, pp. 15654-15663, 2010.

[27] S. Belin, H. Nawabi, C. Wang et al., "Injury-induced decline of intrinsic regenerative ability revealed by quantitative proteomics," Neuron, vol. 86, no. 4, pp. 1000-1014, 2015.

[28] N. Aronin and M. DiFiglia, "Huntingtin-lowering strategies in Huntington's disease: antisense oligonucleotides, small RNAs, and gene editing," Movement Disorders, vol. 29, no. 11, pp. 14551461, 2014.

[29] D. Dalkara and J.-A. Sahel, "Gene therapy for inherited retinal degenerations," Comptes Rendus Biologies, vol. 337, no. 3, pp. 185-192, 2014.
[30] M. Chen, Q. Chen, X. Sun et al., “Generation of retinal ganglionlike cells from reprogrammed mouse fibroblasts," Investigative Ophthalmology and Visual Science, vol. 51, no. 11, pp. 5970-5978, 2010.

[31] B. Jagatha, M. S. Divya, R. Sanalkumar et al., "In vitro differentiation of retinal ganglion-like cells from embryonic stem cell derived neural progenitors," Biochemical and Biophysical Research Communications, vol. 380, no. 2, pp. 230-235, 2009.

[32] S. Parameswaran, S. M. Dravid, P. Teotia et al., "Continuous non-cell autonomous reprogramming to generate retinal ganglion cells for glaucomatous neuropathy," STEM CELLS, vol. 33, no. 6, pp. 1743-1758, 2015.

[33] H. Levkovitch-Verbin, O. Sadan, S. Vander et al., "Intravitreal injections of neurotrophic factors secreting mesenchymal stem cells are neuroprotective in rat eyes following optic nerve transaction," Investigative Ophthalmology and Visual Science, vol. 51, no. 12, pp. 6394-6400, 2010.

[34] C. Zaverucha-do-Valle, F. Gubert, M. Bargas-Rega et al., "Bone marrow mononuclear cells increase retinal ganglion cell survival and axon regeneration in the adult rat," Cell Transplantation, vol. 20, no. 3, pp. 391-406, 2011.

[35] L. A. Mesentier-Louro, C. Zaverucha-do-Valle, A. J. da Silva et al., "Distribution of mesenchymal stem cells and effects on neuronal survival and axon regeneration after optic nerve crush and cell therapy," PLoS ONE, vol. 9, no. 10, Article ID el10722, 2014.

[36] P. H. Rosado-De-Castro, P. M. Pimentel-Coelho, L. M. B. da Fonseca, G. R. de Freitas, and R. Mendez-Otero, "The rise of cell therapy trials for stroke: review of published and registered studies," Stem Cells and Development, vol. 22, no. 15, pp. 20952111, 2013.

[37] R. D. Simari, C. J. Pepine, J. H. Traverse et al., "Bone marrow mononuclear cell therapy for acute myocardial infarction: a perspective from the cardiovascular cell therapy research network," Circulation Research, vol. 114, no. 10, pp. 1564-1568, 2014.

[38] C. Pösel, K. Möller, W. Fröhlich, I. Schulz, J. Boltze, and D.C. Wagner, "Density gradient centrifugation compromises bone marrow mononuclear cell yield," PLoS ONE, vol. 7, no. 12, Article ID e50293, 2012.

[39] E. Zhao, H. Xu, L. Wang et al., "Bone marrow and the control of immunity," Cellular \& Molecular Immunology, vol. 9, no. 1, pp. 11-19, 2012.

[40] W. W. Pang, E. A. Price, D. Sahoo et al., "Human bone marrow hematopoietic stem cells are increased in frequency and myeloid-biased with age," Proceedings of the National Academy of Sciences of the United States of America, vol. 108, no. 50, pp. 20012-20017, 2011.

[41] M. A. Goodell, H. Nguyen, and N. Shroyer, "Somatic stem cell heterogeneity: diversity in the blood, skin and intestinal stem cell compartments," Nature Reviews Molecular Cell Biology, vol. 16, no. 5, pp. 299-309, 2015.

[42] T. Asahara, T. Murohara, A. Sullivan et al., "Isolation of putative progenitor endothelial cells for angiogenesis," Science, vol. 275, no. 5302, pp. 964-967, 1997.

[43] M. D’Aveni, J. Rossignol, T. Coman et al., "G-CSF mobilizes $\mathrm{CD} 4^{+}$regulatory monocytes that inhibit graft-versus-host disease," Science Translational Medicine, vol. 7, no. 281, Article ID 281ra42, 2015.

[44] R. J. Berenson, R. G. Andrews, W. I. Bensinger et al., "Antigen CD34+ marrow cells engraft lethally irradiated baboons," Journal of Clinical Investigation, vol. 81, no. 3, pp. 951-955, 1988. 
[45] Y.-H. Zhao, B. Yuan, J. Chen et al., "Endothelial progenitor cells: therapeutic perspective for ischemic stroke," CNS Neuroscience and Therapeutics, vol. 19, no. 2, pp. 67-75, 2013.

[46] K. Stellos, V. Panagiota, A. Kögel, T. Leyhe, M. Gawaz, and C. Laske, "Predictive value of platelet activation for the rate of cognitive decline in Alzheimer's disease patients," Journal of Cerebral Blood Flow \& Metabolism, vol. 30, no. 11, pp. 1817-1820, 2010.

[47] P. H. Lee, H.-S. Kim, J. E. Lee et al., "Comparison of endothelial progenitor cells in parkinson's disease patients treated with levodopa and levodopa/comt inhibitor," PLOS ONE, vol. 6, no. 6, Article ID e21536, 2011.

[48] M. F. Pittenger, A. M. Mackay, S. C. Beck et al., "Multilineage potential of adult human mesenchymal stem cells," Science, vol. 284, no. 5411, pp. 143-147, 1999.

[49] A. J. Friedenstein, U. F. Deriglasova, N. N. Kulagina et al., "Precursors for fibroblasts in different populations of hematopoietic cells as detected by the in vitro colony assay method," Experimental Hematology, vol. 2, no. 2, pp. 83-92, 1974.

[50] M. Dominici, K. Le Blanc, I. Mueller et al., "Minimal criteria for defining multipotent mesenchymal stromal cells. The International Society for Cellular Therapy position statement," Cytotherapy, vol. 8, no. 4, pp. 315-317, 2006.

[51] J. J. Bara, R. G. Richards, M. Alini, and M. J. Stoddart, "Concise review: bone marrow-derived mesenchymal stem cells change phenotype following in vitro culture: implications for basic research and the clinic," Stem Cells, vol. 32, no. 7, pp. 1713-1723, 2014.

[52] J. Isern and S. Méndez-Ferrer, "Stem cell interactions in a bone marrow niche," Current Osteoporosis Reports, vol. 9, no. 4, pp. 210-218, 2011.

[53] S. Méndez-Ferrer, T. V. Michurina, F. Ferraro et al., "Mesenchymal and haematopoietic stem cells form a unique bone marrow niche," Nature, vol. 466, no. 7308, pp. 829-834, 2010.

[54] L. D. S. Meirelles, A. I. Caplan, and N. B. Nardi, "In search of the in vivo identity of mesenchymal stem cells," Stem Cells, vol. 26, no. 9, pp. 2287-2299, 2008.

[55] C. M. Kolf, E. Cho, and R. S. Tuan, "Mesenchymal stromal cells. Biology of adult mesenchymal stem cells: regulation of niche, self-renewal and differentiation," Arthritis Research and Therapy, vol. 9, no. 1, article 204, 2007.

[56] L. A. Marquez-Curtis, A. Janowska-Wieczorek, L. E. McGann et al., "Cryopreservation of mesenchymal stromal cells derived from various tissues," Cryobiology, vol. 71, no. 2, pp. 181-197, 2015.

[57] B. Rüster, S. Göttig, R. J. Ludwig et al., "Mesenchymal stem cells display coordinated rolling and adhesion behavior on endothelial cells," Blood, vol. 108, no. 12, pp. 3938-3944, 2006.

[58] A. de Becker, P. van Hummelen, M. Bakkus et al., "Migration of culture-expanded human mesenchymal stem cells through bone marrow endothelium is regulated by matrix metalloproteinase- 2 and tissue inhibitor of metalloproteinase3," Haematologica, vol. 92, no. 4, pp. 440-449, 2007.

[59] K. Le Blanc, "Mesenchymal stromal cells: tissue repair and immune modulation," Cytotherapy, vol. 8, no. 6, pp. 559-561, 2006.

[60] D. Kyurkchiev, I. Bochev, E. Ivanova-Todorova, and et al, "Secretion of immunoregulatory cytokines by mesenchymal stem cells," World Journal of Stem Cells, vol. 6, no. 5, pp. 552570, 2014.
[61] T. V. Johnson, N. W. Dekorver, V. A. Levasseur et al., "Identification of retinal ganglion cell neuroprotection conferred by platelet-derived growth factor through analysis of the mesenchymal stem cell secretome," Brain, vol. 137, part 2, pp. 503519, 2014.

[62] B. Mead, A. Logan, M. Berry, W. Leadbeater, and B. A. Scheven, "Paracrine-mediated neuroprotection and neuritogenesis of axotomised retinal ganglion cells by human dental pulp stem cells: comparison with human bone marrow and adiposederived mesenchymal stem cells," PLoS ONE, vol. 9, no. 10, Article ID e109305, 2014.

[63] L. Moraes, A. Vasconcelos-dos-Santos, F. C. Santana et al., "Neuroprotective effects and magnetic resonance imaging of mesenchymal stem cells labeled with SPION in a rat model of Huntington's disease," Stem Cell Research, vol. 9, no. 2, pp. 143155, 2012.

[64] A. D. V. dos Santos, J. da Costa Reis, B. D. Paredes et al., "Therapeutic window for treatment of cortical ischemia with bone marrow-derived cells in rats," Brain Research, vol. 1306, pp. 149-158, 2010.

[65] A. B. Ramos, A. Vasconcelos-Dos-Santos, S. A. L. de Souza et al., "Bone-marrow mononuclear cells reduce neurodegeneration in hippocampal CA1 layer after transient global ischemia in rats," Brain Research, vol. 1522, pp. 1-11, 2013.

[66] H. Wan, F. Li, L. Zhu, J. Wang, Z. Yang, and Y. Pan, "Update on therapeutic mechanism for bone marrow stromal cells in ischemic stroke," Journal of Molecular Neuroscience, vol. 52, no. 2, pp. 177-185, 2014.

[67] S. Agadi and A. K. Shetty, "Concise review: prospects of bone marrow mononuclear cells and mesenchymal stem cells for treating status epilepticus and chronic epilepsy," Stem Cells, vol. 33, no. 7, pp. 2093-2103, 2015.

[68] E. Emre, N. Yüksel, G. Duruksu et al., "Neuroprotective effects of intravitreally transplanted adipose tissue and bone marrowderived mesenchymal stem cells in an experimental ocular hypertension model," Cytotherapy, vol. 17, no. 5, pp. 543-559, 2015.

[69] S. Yu, T. Tanabe, M. Dezawa, H. Ishikawa, and N. Yoshimura, "Effects of bone marrow stromal cell injection in an experimental glaucoma model," Biochemical and Biophysical Research Communications, vol. 344, no. 4, pp. 1071-1079, 2006.

[70] M. M. Harper, S. D. Grozdanic, B. Blits et al., “Transplantation of BDNF-secreting mesenchymal stem cells provides neuroprotection in chronically hypertensive rat eyes," Investigative Ophthalmology \& Visual Science, vol. 52, no. 7, pp. 4506-4515, 2011.

[71] T. V. Johnson, N. D. Bull, D. P. Hunt, N. Marina, S. I. Tomarev, and K. R. Martin, "Neuroprotective effects of intravitreal mesenchymal stem cell transplantation in experimental glaucoma," Investigative Ophthalmology and Visual Science, vol. 51, no. 4, pp. 2051-2059, 2010.

[72] N. Li, X. R. Li, and J. Q. Yuan, "Effects of bone-marrow mesenchymal stem cells transplanted into vitreous cavity of rat injured by ischemia/reperfusion," Graefe's Archive for Clinical and Experimental Ophthalmology, vol. 247, no. 4, pp. 503-514, 2009.

[73] Y. Hu, H. B. Tan, X. M. Wang, H. Rong, H. P. Cui, and H. Cui, "Bone marrow mesenchymal stem cells protect against retinal ganglion cell loss in aged rats with glaucoma," Clinical Interventions in Aging, vol. 8, pp. 1467-1470, 2013. 
[74] R. Manuguerra-Gagné, P. R. Boulos, A. Ammar et al., “Transplantation of mesenchymal stem cells promotes tissue regeneration in a glaucoma model through laser-induced paracrine factor secretion and progenitor cell recruitment," Stem Cells, vol. 31, no. 6, pp. 1136-1148, 2013.

[75] L. A. Mesentier-Louro, J. Coronel, C. Zaverucha-do-Valle et al., "Cell therapy modulates expression of Taxl-binding protein 1 and synaptotagmin IV in a model of optic nerve lesion," Investigative Ophthalmology and Visual Science, vol. 53, no. 8, pp. 4720-4729, 2012.

[76] C. Zaverucha-do-Valle, L. Mesentier-Louro, F. Gubert et al., "Sustained effect of bone marrow mononuclear cell therapy in axonal regeneration in a model of optic nerve crush," Brain Research, vol. 1587, no. 1, pp. 54-68, 2014.

[77] H. B. Tan, X. Kang, S. H. Lu, and L. Liu, "The therapeutic effects of bone marrow mesenchymal stem cells after optic nerve damage in the adult rat," Clinical Interventions in Aging, vol. 10, pp. 487-490, 2015.

[78] T. Zhao, Y. Li, L. Tang, Y. Li, F. Fan, and B. Jiang, "Protective effects of human umbilical cord blood stem cell intravitreal transplantation against optic nerve injury in rats," Graefe's Archive for Clinical and Experimental Ophthalmology, vol. 249, no. 7, pp. 1021-1028, 2011.

[79] B. Jiang, P. Zhang, D. Zhou, J. Zhang, X. Xu, and L. Tang, "Intravitreal transplantation of human umbilical cord blood stem cells protects rats from traumatic optic neuropathy," PLoS ONE, vol. 8, no. 8, Article ID e69938, 2013.

[80] M. Chen, Z. Xiang, and J. Cai, “The anti-apoptotic and neuroprotective effects of human umbilical cord blood mesenchymal stem cells (hUCB-MSCs) on acute optic nerve injury is transient," Brain Research, vol. 1532, pp. 63-75, 2013.

[81] L. M. Carlin, E. G. Stamatiades, C. Auffray et al., "Nr4aldependent Ly6C $\mathrm{C}^{\text {low }}$ monocytes monitor endothelial cells and orchestrate their disposal," Cell, vol. 153, no. 2, pp. 362-375, 2013.

[82] F. Ginhoux and S. Jung, "Monocytes and macrophages: developmental pathways and tissue homeostasis," Nature Reviews Immunology, vol. 14, no. 6, pp. 392-404, 2014.

[83] A. London, E. Itskovich, I. Benhar et al., "Neuroprotection and progenitor cell renewal in the injured adult murine retina requires healing monocyte-derived macrophages," The Journal of Experimental Medicine, vol. 208, no. 1, pp. 23-39, 2011.

[84] T. F. Yuan, Y. X. Liang, B. Peng, B. Lin, and K. So, "Local proliferation is the main source of rod microglia after optic nerve transection," Scientific Reports, vol. 5, Article ID 10788, 2015.

[85] S. Leon, Y. Yin, J. Nguyen, N. Irwin, and L. I. Benowitz, "Lens injury stimulates axon regeneration in the mature rat optic nerve," Journal of Neuroscience, vol. 20, no. 12, pp. 4615-4626, 2000.

[86] T. Kurimoto, Y. Yin, G. Habboub et al., "Neutrophils express oncomodulin and promote optic nerve regeneration," The Journal of Neuroscience, vol. 33, no. 37, pp. 14816-14824, 2013.

[87] Y. Yin, Q. Cui, H.-Y. Gilbert et al., "Oncomodulin links inflammation to optic nerve regeneration," Proceedings of the National Academy of Sciences of the United States of America, vol. 106, no. 46, pp. 19587-19592, 2009.

[88] Y. Huang, Z. Li, N. van Rooijen, N. Wang, C. P. Pang, and Q. Cui, "Different responses of macrophages in retinal ganglion cell survival after acute ocular hypertension in rats with different autoimmune backgrounds," Experimental Eye Research, vol. 85, no. 5, pp. 659-666, 2007.
[89] I. N. Shalova, J. Y. Lim, M. Chittezhath et al., "Human monocytes undergo functional re-programming during sepsis mediated by hypoxia-inducible factor-1 $\alpha$," Immunity, vol. 42 , no. 3, pp. 484-498, 2015.

[90] A. Yanai, U. O. Häfeli, A. L. Metcalfe et al., "Focused magnetic stem cell targeting to the retina using superparamagnetic iron oxide nanoparticles," Cell Transplantation, vol. 21, no. 6, pp. 1137-1148, 2012.

[91] T. V. Johnson, N. D. Bull, and K. R. Martin, "Identification of barriers to retinal engraftment of transplanted stem cells," Investigative Ophthalmology and Visual Science, vol. 51, no. 2, pp. 960-970, 2010.

[92] P. Castanheira, L. Torquetti, M. B. Nehemy, and A. M. Goes, "Retinal incorporation and differentiation of mesenchymal stem cells intravitreally injected in the injured retina of rats," Arquivos Brasileiros de Oftalmologia, vol. 71, no. 5, pp. 644-650, 2008.

[93] P. Castanheira, L. T. Torquetti, D. R. S. Magalhãs, M. B. Nehemy, and A. M. Goes, "DAPI diffusion after intravitreal injection of mesenchymal stem cells in the injured retina of rats," Cell Transplantation, vol. 18, no. 4, pp. 423-431, 2009.

[94] M. S. Singh and R. E. MacLaren, "Stem cells as a therapeutic tool for the blind: biology and future prospects," Proceedings of the Royal Society B: Biological Sciences, vol. 278, no. 1721, pp. 30093016, 2011.

[95] P. A. Keane and S. R. Sadda, "Retinal imaging in the twenty-first century," Ophthalmology, vol. 121, no. 12, pp. 2489-2500, 2014.

[96] A. S. Arbab, B. Janic, J. Haller, E. Pawelczyk, W. Liu, and J. A. Frank, "In vivo cellular imaging for translational medical research," Current Medical Imaging Reviews, vol. 5, no. 1, pp. 1938, 2009.

[97] M. D. Fischer, T. Goldmann, C. Wallrapp et al., "Successful subretinal delivery and monitoring of MicroBeads in mice," PLoS ONE, vol. 8, no. 1, Article ID e55173, 2013.

[98] T. W. Corson, B. C. Samuels, A. A. Wenzel et al., "Multimodality imaging methods for assessing retinoblastoma orthotopic xenograft growth and development," PLoS ONE, vol. 9, no. 6, Article ID e99036, 2014.

[99] S. S. Yaghoubi, D. O. Campbell, C. G. Radu, and J. Czernin, "Positron emission tomography reporter genes and reporter probes: gene and cell therapy applications," Theranostics, vol. 2, no. 4, pp. 374-391, 2012.

[100] D. L. Kraitchman and J. W. M. Bulte, "Imaging of stem cells using MRI," Basic Research in Cardiology, vol. 103, no. 2, pp. 105113, 2008.

[101] C. J. Palestro, C. Love, and K. K. Bhargava, "Labeled leukocyte imaging: current status and future directions," Quarterly Journal of Nuclear Medicine and Molecular Imaging, vol. 53, no. 1, pp. 105-123, 2009.

[102] G. Mariani, L. Bruselli, T. Kuwert et al., "A review on the clinical uses of SPECT/CT," European Journal of Nuclear Medicine and Molecular Imaging, vol. 37, no. 10, pp. 1959-1985, 2010.

[103] A. Bockisch, L. S. Freudenberg, D. Schmidt, and T. Kuwert, "Hybrid imaging by SPECT/CT and PET/CT: proven outcomes in cancer imaging," Seminars in Nuclear Medicine, vol. 39, no. 4, pp. 276-289, 2009.

[104] D. L. Kraitchman, W. D. Gilson, and C. H. Lorenz, "Stem cell therapy: MRI guidance and monitoring," Journal of Magnetic Resonance Imaging, vol. 27, no. 2, pp. 299-310, 2008.

[105] A. Haddad-Mashadrizeh, A. R. Bahrami, M. M. Matin et al., "Human adipose-derived mesenchymal stem cells can survive 
and integrate into the adult rat eye following xenotransplantation," Xenotransplantation, vol. 20, no. 3, pp. 165-176, 2013.

[106] B. Mead, A. Logan, M. Berry, W. Leadbeater, and B. A. Scheven, "Intravitreally transplanted dental pulp stem cells promote neuroprotection and axon regeneration of retinal ganglion cells after optic nerve injury," Investigative Ophthalmology and Visual Science, vol. 54, no. 12, pp. 7544-7556, 2013.

[107] M. Fiedorowicz, W. Dyda, R. Rejdak, and P. Grieb, "Magnetic resonance in studies of glaucoma," Medical Science Monitor, vol. 17, no. 10, pp. RA227-RA232, 2011.

[108] R. Haenold, K.-H. Herrmann, S. Schmidt et al., "Magnetic resonance imaging of the mouse visual pathway for in vivo studies of degeneration and regeneration in the CNS," NeuroImage, vol. 59, no. 1, pp. 363-376, 2012.

[109] T. Watanabe, O. Natt, S. Boretius, J. Frahm, and T. Michaelis, "In vivo 3D MRI staining of mouse brain after subcutaneous application of $\mathrm{MnCl}_{2}$," Magnetic Resonance in Medicine, vol. 48, no. 5, pp. 852-859, 2002.

[110] B. A. Berkowitz, R. Roberts, H. Luan et al., "Manganeseenhanced MRI studies of alterations of intraretinal ion demand in models of ocular injury," Investigative Ophthalmology and Visual Science, vol. 48, no. 8, pp. 3796-3804, 2007.

[111] S. Boretius, I. Gadjanski, I. Demmer et al., "MRI of optic neuritis in a rat model," NeuroImage, vol. 41, no. 2, pp. 323-334, 2008.

[112] R. G. Pautler and A. P. Koretsky, "Tracing odor-induced activation in the olfactory bulbs of mice using manganese-enhanced magnetic resonance imaging," NeuroImage, vol. 16, no. 2, pp. 441-448, 2002.

[113] M. Thuen, M. Berry, T. B. Pedersen et al., "Manganese-enhanced MRI of the rat visual pathway: acute neural toxicity, contrast enhancement, axon resolution, axonal transport, and clearance of $\mathrm{Mn}^{2+}$," Journal of Magnetic Resonance Imaging, vol. 28, no. 4, pp. 855-865, 2008.

[114] T.-H. Lin, C.-W. Chiang, K. Trinkaus, W. M. Spees, P. Sun, and S.-K. Song, "Manganese-enhanced MRI (MEMRI) via topical loading of $\mathrm{Mn}^{2+}$ significantly impairs mouse visual acuity: a comparison with intravitreal injection," NMR in Biomedicine, vol. 27, no. 4, pp. 390-398, 2014.

[115] A. Kretz, C. J. Happold, J. K. Marticke, and S. Isenmann, "Erythropoietin promotes regeneration of adult CNS neurons via Jak2/Stat3 and PI3K/AKT pathway activation," Molecular and Cellular Neuroscience, vol. 29, no. 4, pp. 569-579, 2005.

[116] F. C. Mansergh, N. Chadderton, P. F. Kenna, O. L. Gobbo, and G. J. Farrar, "Cell therapy using retinal progenitor cells shows therapeutic effect in a chemically-induced rotenone mouse model of Leber hereditary optic neuropathy," European Journal of Human Genetics, vol. 22, no. 11, pp. 1314-1320, 2014.

[117] J. Crossgrove and W. Zheng, "Manganese toxicity upon overexposure," NMR in Biomedicine, vol. 17, no. 8, pp. 544-553, 2004.

[118] X. Zhang, P. Sun, J. Wang, Q. Wang, and S.-K. Song, "Diffusion tensor imaging detects retinal ganglion cell axon damage in the mouse model of optic nerve crush," Investigative Ophthalmology and Visual Science, vol. 52, no. 9, pp. 7001-7006, 2011.

[119] M. Thuen, Ø. Olsen, M. Berry et al., "Combination of $\mathrm{Mn}^{2+}$ enhanced and diffusion tensor MR imaging gives complementary information about injury and regeneration in the adult rat optic nerve," Journal of Magnetic Resonance Imaging, vol. 29, no. 1, pp. 39-51, 2009.

[120] S. L. Grillo and P. Koulen, "Psychophysical testing in rodent models of glaucomatous optic neuropathy," Experimental Eye Research, 2015.
[121] B. Young, E. Eggenberger, and D. Kaufman, "Current electrophysiology in ophthalmology: a review," Current Opinion in Ophthalmology, vol. 23, no. 6, pp. 497-505, 2012.

[122] Z.-J. Zhang, Y.-J. Li, X.-G. Liu et al., "Human umbilical cord blood stem cells and brain-derived neurotrophic factor for optic nerve injury: a biomechanical evaluation," Neural Regeneration Research, vol. 10, no. 7, p. 1134, 2015.

[123] I. B. Black and D. Woodbury, "Adult rat and human bone marrow stromal stem cells differentiate into neurons," Blood Cells, Molecules, and Diseases, vol. 27, no. 3, pp. 632-636, 2001.

[124] W. Deng, M. Obrocka, I. Fischer, and D. J. Prockop, "In vitro differentiation of human marrow stromal cells into early progenitors of neural cells by conditions that increase intracellular cyclic AMP," Biochemical and Biophysical Research Communications, vol. 282, no. 1, pp. 148-152, 2001.

[125] G. Muñoz-Elias, D. Woodbury, and I. B. Black, "Marrow stromal cells, mitosis, and neuronal differentiation: stem cell and precursor functions," Stem Cells, vol. 21, no. 4, pp. 437-448, 2003.

[126] D. Woodbury, E. J. Schwarz, D. J. Prockop, and I. B. Black, "Adult rat and human bone marrow stromal cells differentiate into neurons," Journal of Neuroscience Research, vol. 61, no. 4, pp. 364-370, 2000.

[127] M. Alvarez-Dolado, R. Pardal, J. M. Garcia-Verdugo et al., "Fusion of bone-marrow-derived cells with Purkinje neurons, cardiomyocytes and hepatocytes," Nature, vol. 425, no. 6961, pp. 968-973, 2003.

[128] A. M. Parr, C. H. Tator, and A. Keating, "Bone marrow-derived mesenchymal stromal cells for the repair of central nervous system injury," Bone Marrow Transplantation, vol. 40, no. 7, pp. 609-619, 2007.

[129] N. Terada, T. Hamazaki, M. Oka et al., "Bone marrow cells adopt the phenotype of other cells by spontaneous cell fusion," Nature, vol. 416, no. 6880, pp. 542-545, 2002.

[130] A. Uccelli, L. Moretta, and V. Pistoia, "Mesenchymal stem cells in health and disease," Nature Reviews Immunology, vol. 8, no. 9, pp. 726-736, 2008.

[131] N. Nakano, Y. Nakai, T.-B. Seo et al., "Characterization of conditioned medium of cultured bone marrow stromal cells," Neuroscience Letters, vol. 483, no. 1, pp. 57-61, 2010.

[132] A. Tassoni, A. Gutteridge, A. C. Barber, A. Osborne, and K. R. Martin, "Molecular mechanisms mediating retinal reactive gliosis following bone marrow mesenchymal stem cell (BMMSC) transplantation," Stem Cells, 2015.

[133] G. M. Spaggiari and L. Moretta, "Cellular and molecular interactions of mesenchymal stem cells in innate immunity," Immunology and Cell Biology, vol. 91, no. 1, pp. 27-31, 2013.

[134] G. Raicevic, R. Rouas, M. Najar et al., "Inflammation modifies the pattern and the function of Toll-like receptors expressed by human mesenchymal stromal cells," Human Immunology, vol. 71, no. 3, pp. 235-244, 2010.

[135] R. S. Waterman, S. L. Tomchuck, S. L. Henkle, and A. M. Betancourt, "A new mesenchymal stem cell (MSC) paradigm: polarization into a pro-inflammatory $\mathrm{MSC1}$ or an immunosuppressive MSC2 phenotype," PLoS ONE, vol. 5, no. 4, Article ID e10088, 2010.

[136] T. Katsuda, N. Kosaka, F. Takeshita, and T. Ochiya, "The therapeutic potential of mesenchymal stem cell-derived extracellular vesicles," Proteomics, vol. 13, no. 10-11, pp. 1637-1653, 2013.

[137] D. Giunti, B. Parodi, C. Usai et al., "Mesenchymal stem cells shape microglia effector functions through the release of CX3CL1," Stem Cells, vol. 30, no. 9, pp. 2044-2053, 2012. 
[138] D. Drago, C. Cossetti, N. Iraci et al., "The stem cell secretome and its role in brain repair," Biochimie, vol. 95, no. 12, pp. 22712285, 2013.

[139] H. Xin, Y. Li, Z. Liu et al., "miR-133b promotes neural plasticity and functional recovery after treatment of stroke with multipotent mesenchymal stromal cells in rats via transfer of exosomeenriched extracellular particles," Stem Cells, vol. 31, no. 12, pp. 2737-2746, 2013.

[140] J. B. Jonas, M. Witzens-Harig, L. Arseniev, and A. D. Ho, "Intravitreal autologous bone marrow-derived mononuclear cell transplantation: a feasibility report," Acta ophthalmologica, vol. 86, no. 2, pp. 225-226, 2008.

[141] P. Connick, M. Kolappan, C. Crawley et al., "Autologous mesenchymal stem cells for the treatment of secondary progressive multiple sclerosis: an open-label phase $2 \mathrm{a}$ proof-of-concept study," The Lancet Neurology, vol. 11, no. 2, pp. 150-156, 2012.

[142] B. Mead, M. Berry, A. Logan, R. A. Scott, W. Leadbeater, and B. A. Scheven, "Stem cell treatment of degenerative eye disease," Stem Cell Research, vol. 14, no. 3, pp. 243-257, 2015. 

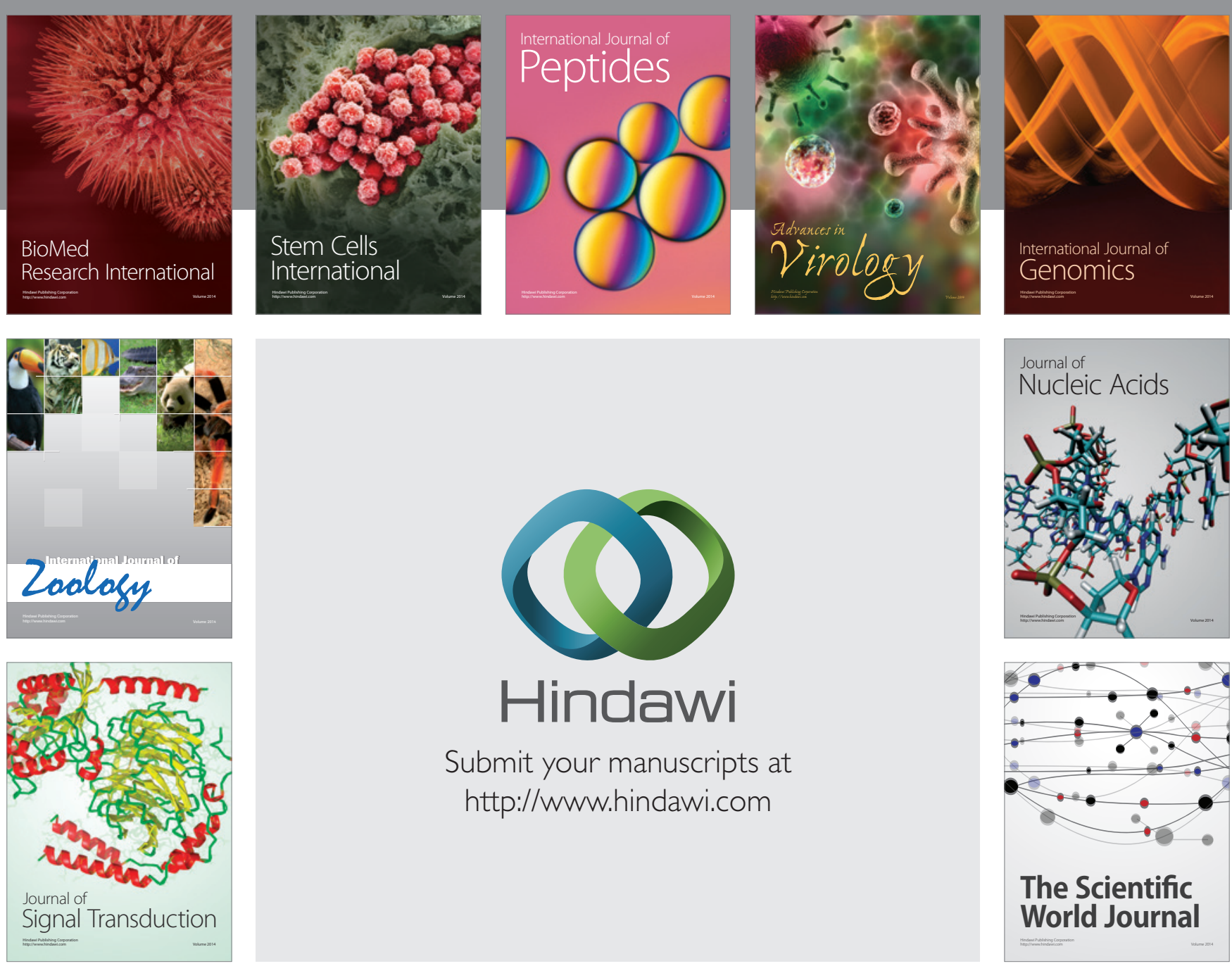

Submit your manuscripts at

http://www.hindawi.com
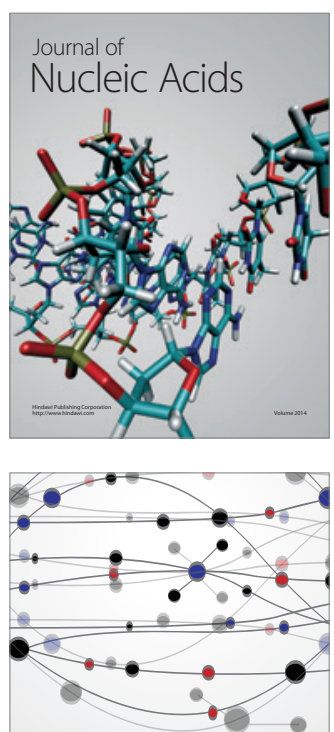

The Scientific World Journal
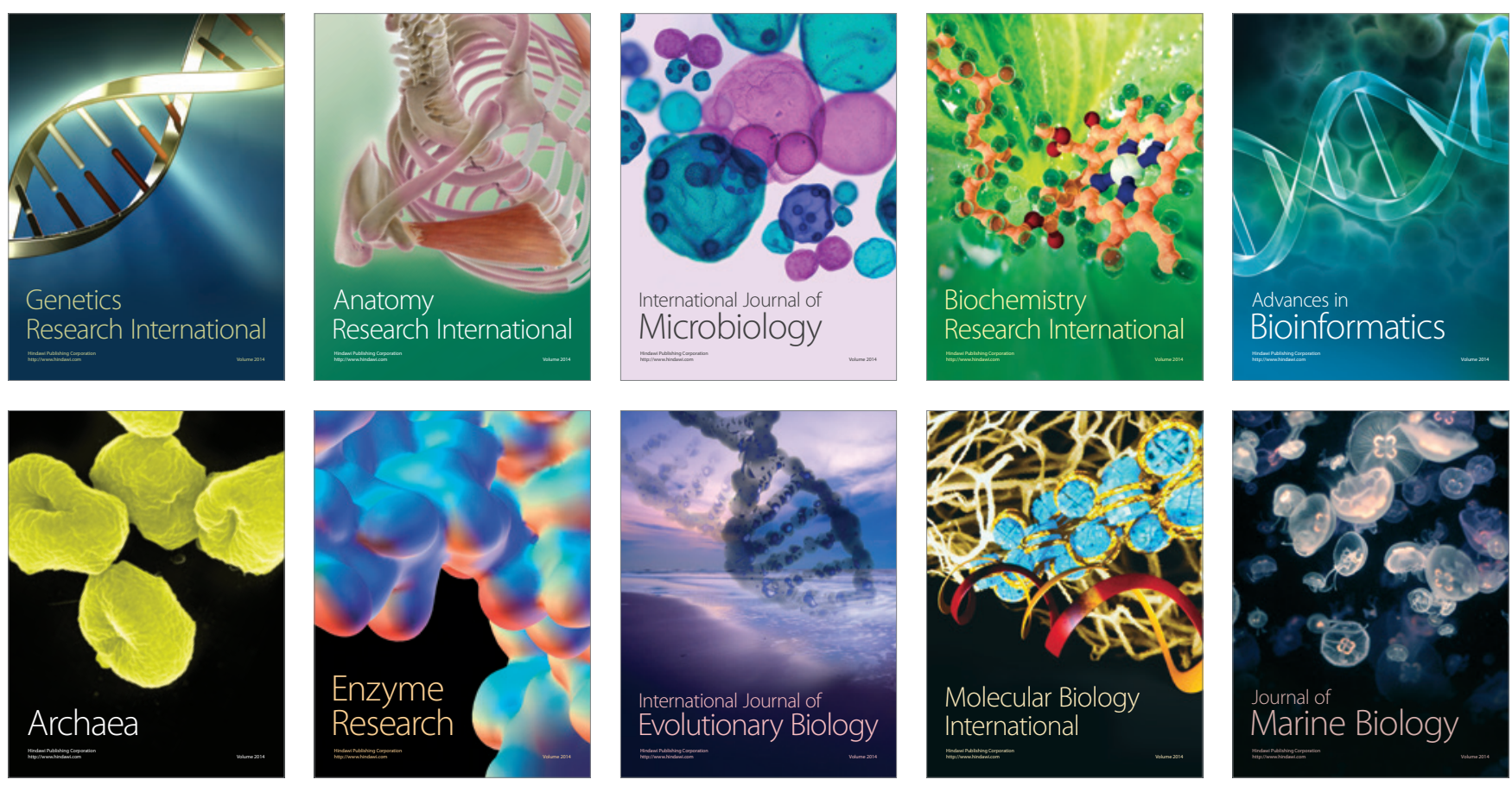